\title{
Avaliação de riscos ocupacionais de motorista de ônibus intermunicipal: um estudo de caso no Estado do Pará
}

\author{
Wanderson Lyrio Bermudes \\ Engenheiro de Produção (UNIVERSO), Engenheiro de Segurança do Trabalho (UFF), Mestre em Tecnologia \\ Ambiental (FAACZ) e Doutor em Ciências Florestais (UFES). Professor do IFES - Campus Vitória - ES \\ 凶wanderson.bermudes@hotmail.com
}

\section{Luciano José Minette}

Engenheiro Florestal pela Universidade Federal de Viçosa (UFV), Engenharia de Segurança do Trabalho - FUMEC, Mestrado em Ciência Florestal pela Universidade Federal de Viçosa (UFV), Doutorado em Ciência Florestal pela Universidade Federal de Viçosa (UFV). Professor Titular da Universidade Federal de Viçosa (UFV), integrante da Universidade Federal do Espírito Santo (UFES)

$凶$ minette@ufv.br

\section{José Rodrigo Cunha}

Engenheiro Florestal (2009), Engenharia de Segurança do Trabalho (2013) e MBA Gestão de Pessoa (2016). Coordenador de SESMT área Industrial e Florestal nos estados do Pará e Amapá

Recebido em 5 de abril de 2019

Aceito em 30 de maio de 2019

\begin{abstract}
Resumo:
O transporte urbano é relevante para o deslocamento de pessoas, acessibilidade e permite a integração de diversas áreas econômicas. Apesar da importância, o condutor do veículo nem sempre possui boas condições de conforto, saúde e segurança, tendo no período de 2006 a 2017, no Brasil, o registro de 103.231 acidentes de trabalho com trabalhadores que realizam o transporte urbano com itinerário fixo, intermunicipal, região metropolitana, interestadual e internacional. Diante desse cenário, essa pesquisa tem por objetivo avaliar os riscos físicos ruído e vibração de corpo inteiro e o ergonômico para a função de motorista no norte do estado do Pará. Para a realização da avaliação do ruído e vibração foram adotadas as metodologias das normas de higiene ocupacional 01 e 09 da Fundação Jorge Duprat e Figueiredo e as normas regulamentadoras 09 e 15 do governo federal, para a avaliação do risco ergonômico foi utilizada a metodologia Rapid Entire Body Assessment. Os resultados identificaram o risco de ruído de até $82 \mathrm{~dB}(\mathrm{~A})$ e a vibração de corpo inteiro máxima de 10,20 $\mathrm{m} . \mathrm{s}^{-1,75}$ resultados esses inferiores ao limite de tolerância, porém superior ao nível de ação, que indica a necessidade de adoção de medidas de controle. O risco ergonômico foi avaliado como médio, sendo também recomendadas ações de controle. Para controle do risco, pesquisas sugerem a manutenção periódica dos veículos, substituição do sistema de suspensão e inclusão do câmbio automático, de modo a tornar o ambiente veicular mais seguros, confortáveis e modernos, compatíveis com a relevância do trabalho.
\end{abstract}

Palavras-chave: Transporte, Condutor, Ambiente, Segurança. 
Avaliação de riscos ocupacionais de motorista de ônibus

intermunicipal: um estudo de caso no Estado do Pará

\title{
Evaluation of occupational risks of intercity bus drivers: a case study in the State of Pará
}

\begin{abstract}
:
Urban transport is relevant to the movement of people, accessibility and allows the integration of several economic areas. Despite the importance, the driver of the vehicle does not always have good conditions of comfort, health and safety, having in the period from 2006 to 2017, in Brazil, the registration of 103,231 work accidents with workers who carry out urban transportation with fixed, intermunicipal itinerary, metropolitan region, interstate and international. Considering this scenario, this research aims to evaluate the physical risks of noise and vibration of the whole body and the ergonomic for the function of driver in the north of the state of Pará. In order to perform noise and vibration evaluation, the methodologies of the standards of occupational hygiene 01 and 09 of the Jorge Duprat and Figueiredo Foundation, and the Federal Government regulations 09 and 15, the Rapid Entire Body Assessment methodology was used to evaluate the ergonomic risk. The results identified the noise risk of up to $82 \mathrm{~dB}(\mathrm{~A})$ and maximum body vibration of $10.20 \mathrm{~m} \cdot \mathrm{s}^{-1.75}$ results that are below the tolerance limit, but above the action level, indicating the need for adoption of control measures. The ergonomic risk was evaluated as medium, and control actions were also recommended. For risk control, surveys suggest periodic maintenance of vehicles, replacement of the suspension system and inclusion of the automatic gearbox, in order to make the vehicle environment safer, comfortable and modern, compatible with the relevance of the work.

Keywords: Transportation, Conductor, Environment, Safety.
\end{abstract}

\section{Evaluación de riesgos ocupacionales de conductor de autobús intermunicipal: un estudio de caso en el Estado de Pará}

\section{Resumen:}

El transporte urbano es relevante para el desplazamiento de personas, accesibilidad y permite la integración de diversas áreas económicas. A pesar de la importancia, el conductor del vehículo no siempre tiene buenas condiciones de confort, salud y seguridad, teniendo en el período de 2006 a 2017, en Brasil, el registro de 103.231 accidentes de trabajo con trabajadores que realizan el transporte urbano con itinerario fijo, intermunicipal, región metropolitana, interestatal e internacional. En este escenario, esta investigación tiene por objetivo evaluar los riesgos físicos de ruido y vibración de cuerpo entero y el ergonómico para la función de conductor en el norte del estado de Pará. Para la realización de la evaluación del ruido y la vibración se adoptaron las metodologías de las normas la higiene ocupacional 01 y 09 de la Fundación Jorge Duprat y Figueiredo y las normas reguladoras 09 y 15 del gobierno federal, para la evaluación del riesgo ergonómico fue utilizada la metodología Rapid Entire Body Assessment. Los resultados identificaron el riesgo de ruido de hasta $82 \mathrm{~dB}$ (A) y la vibración de cuerpo entero máxima de $10,20 \mathrm{~ms}^{-1,75}$ resultados inferiores al límite de tolerancia, pero superior al nivel de acción, que indica la necesidad de la adopción de medidas de control. El riesgo ergonómico fue evaluado como medio, siendo también recomendadas acciones de control. Para el control del riesgo, las investigaciones sugieren el mantenimiento periódico de los vehículos, la sustitución del sistema de suspensión e inclusión del cambio automático, para que el ambiente sea más seguro, cómodo y moderno, compatible con la relevancia del trabajo.

Palabras clave: Transporte, Conductor, Medio ambiente, Seguridad. 


\section{INTRODUÇÃO}

O transporte urbano é essencial para as pessoas, e necessário meio de integração entre as diversas áreas econômicas, com atribuição de promover acessibilidade, mobilidade e qualidade de vida (SILVA; SCHLAG, 2017).

No tocante a qualidade do serviço prestado no transporte urbano, nem sempre ele está a contento para o usuário a uma tarifa justa e adequado a saúde e segurança do condutor do veículo (SILVA, J.; SILVA, S., 2018).

O motorista tem sua condição de saúde afetada seja pelos fatores ambientais ou a interação social que provoca o aumento do estresse, sendo que ele é o responsável direto pelo veículo conduzido, pela integridade física dos passageiros e pela própria imagem da empresa (ALQUIMIM et al., 2018; BATTISTON; CRUZ; HOFFMANN, 2006).

No exercício da sua função, o motorista depara-se com variadas situações com potencial estressor, a precariedade do asfalto, baixos salários, a condição dos veículos, ruídos, calor, vibração, congestionamento, a relação com o público e a insegurança (exposto a assalto), contribuindo para o aparecimento de doenças, acidentes de trânsito e de trabalho (ALQUIMIM et al., 2018).

Moraes et al (2018) retrata essa precariedade no Estado do Pará, ao verificar a opinião dos usuários do transporte urbano, observou em sua pesquisa a falha no conforto durante a viagem, na limpeza nos ônibus e nos terminais e o risco elevado de assaltos. Matos e Neves (2015) descrevem os riscos observados pelos motoristas ao desempenharem suas atividades e destacam o ruído, a vibração, posturas incomodas, o trânsito e as relações interpessoais com os passageiros como agentes causadores de estresse e acidentes.

Esses riscos contribuíram para a ocorrência de 103.231 acidentes de trabalho no período de 2006 a 2017 no Brasil, nas atividades de transporte urbano com itinerário fixo, intermunicipal, região metropolitana, interestadual e internacional, somados com os acidentes ocorridos durante o deslocamento do trabalho para casa e vice-versa desses trabalhadores. Considerando apenas o Estado do Pará foram notificados 1.657 acidentes conforme o Anuário Estatístico de Acidente do Trabalho - AEAT (2017).

Dentre os fatores de risco destaca os ambientais (físicos: ruído e vibração de corpo inteiro), conforme apresentado nas Normas Regulamentadoras - NR 09 e 15 e o risco ergonômico (postura) abordado na NR 17. 
Diante da relevância da atividade de condutor do transporte urbano e dos riscos associados a esta atividade, a pesquisa tem por objetivo avaliar as condições ambientais (risco físico - ruído e vibração) e ergonômicas (postura) do motorista realizadas no Estado do Pará, de forma a contribuir com ações de prevenção e controle de risco, quando necessárias.

\section{METODOLOGIA}

As avaliações, descritas nessa pesquisa, foram realizadas em julho de 2017 em um ônibus que realizava o transporte urbano de trabalhadores intermunicipal no norte do Estado do Pará, localizado no norte do Brasil, em vias que intercalavam asfalto e estrada de terra em dia com baixa nebulosidade (FAPESPA, 2016; MORAES et al., 2005). O veículo utilizado na avaliação possui ar condicionado, câmbio manual e o motor é localizado na dianteira.

Para realização da avaliação de ruído foi utilizada a metodologia da Norma de Higiene Ocupacional - NHO 01 e o resultado comparado com o Limite de Tolerância - LT da Norma Regulamentadora - NR 15 que é de $85 \mathrm{~dB}$ (A) e o nível de ação de $80 \mathrm{~dB}(\mathrm{~A})$ para uma jornada padrão de oito horas conforme NR - 09 (ATLAS, 2019; FUNDACENTRO, 2001). As avaliações de ruído cobriram toda a jornada de trabalho que é de 06 horas (06h:00min às 12h:00min (diurno) e 12h:00min às 18h:00min (vespertino)) e o resultado projetado para uma jornada padrão de oito horas (FUNDACENTRO, 2001).

Essa avaliação foi realizada com o audiodosimetro Edge série ESM 070152 e cobriu todo o ciclo de exposição, com o aparelho configurado seguindo os seguintes critérios: fator de duplicação de dose igual a 5, dose de $100 \%$ para o limite de tolerância de $85 \mathrm{~dB}(\mathrm{~A})$, nível limiar de integração de $80 \mathrm{~dB}(\mathrm{~A})$, curva de ponderação (A) e circuito de resposta Slow, com o microfone posicionado na zona auditiva do trabalhador.

No que se refere à avaliação de vibração de corpo inteiro foi adotado a método da NHO 09 e NR 15 com a utilização de um medidor de vibração de 04 canais, modelo Maestro, fabricante $01 \mathrm{~dB}$ do Brasil, $\mathrm{n}^{\circ}$ de série 12547, com o acelerômetro posicionado de acordo com os eixos x, y e z (Figura 1) (FUNDACENTRO, 2013). 
Figura 1. Posicionamento do acelerômetro para avaliação de vibração de corpo inteiro

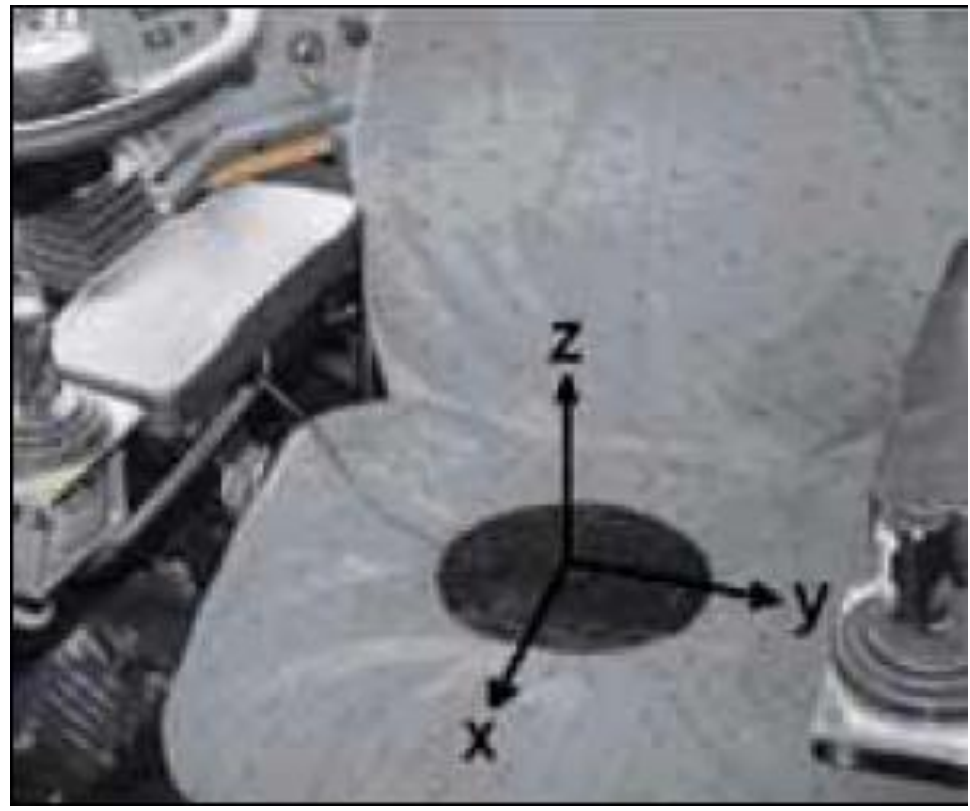

Fonte: FUNDACENTRO, 2013.

O Limite de tolerância para vibração de corpo inteiro é para a avaliação em aceleração de $1,1 \mathrm{~m} \cdot \mathrm{s}^{-2}$ e 21,0 $\mathrm{m} \cdot \mathrm{s}^{-1,75}$ para o Valor de Dose de Vibração - VDV e o nível de ação de $0,5 \mathrm{~m} \cdot \mathrm{s}^{-2}$ e $9,1 \mathrm{~m} \cdot \mathrm{s}^{-1,75}$ respectivamente, projetado para uma jornada padrão de oito horas.

$\mathrm{Na}$ avaliação ergonômica do posto de trabalho foi adotada a metodologia da Rapid Entire Body Assessment - REBA desenvolvido por Mcatamney e Hignett, (2000), que avalia a atividade muscular estática adotada pelo corpo humano.

A REBA é uma ferramenta semiquantitativa usada para avaliar o risco de distúrbio musculoesquelético a partir da análise postural dos trabalhadores. O objetivo da avaliação é analisar as posturas e enquadrá-las conforme níveis de risco e categoria de ação, para resolução dos problemas diagnosticados (MCATAMNEY; HIGNETT, 2000).

A codificação das regiões corporais é estabelecida por diagramas representativos associados a tabela de pontos divididos em dois grupos: A e B (Quadro 1) (MCATAMNEY; HIGNETT, 2000).

Os diagramas do Grupo A (Pescoço, Tronco e Pernas) são compostos por um total de 60 combinações de posturas, o que resulta em um total de 10 possíveis pontos encontrados no Quadro 2 que serão somados ao ponto de carga/força (CARDOSO JUNIOR, 2006). 
Quadro 1 - Classificação dos grupos e segmentos corpóreos avaliados

\begin{tabular}{|c|c|}
\hline Grupo & Segmento Corpóreo Avaliado \\
\hline A & Pescoço, Troco e Pernas \\
\hline B & Braços, Antebraços e Punhos \\
\hline
\end{tabular}

Fonte: MCATAMNEY; HIGNETT, 2000.

A postura do tronco tem pontuação que varia de 1 a 5, conforme posição (1- tronco erguido; 2- tronco com flexão entre $0^{\circ}$ a $20^{\circ}$; 3- tronco com flexão entre $20^{\circ}$ e $60^{\circ}$; 4- tronco com flexão superior a $60^{\circ}$ e deve ser acrescido 1 ponto casa haja torção ou flexão lateral do tronco.

Quadro 2 - Pontuações do Grupo A somados ao escore carga/força

\begin{tabular}{|c|c|c|c|c|c|c|c|c|c|c|c|c|}
\hline \multirow{4}{*}{ Tronco } & \multicolumn{12}{|c|}{ Pescoço } \\
\hline & \multicolumn{4}{|c|}{1} & \multicolumn{4}{|c|}{2} & \multicolumn{4}{|c|}{3} \\
\hline & \multicolumn{12}{|c|}{ Pernas } \\
\hline & 1 & 2 & 3 & 4 & 1 & 2 & 3 & 4 & 1 & 2 & 3 & 4 \\
\hline 1 & 1 & 2 & 3 & 4 & 1 & 2 & 3 & 4 & 3 & 3 & 5 & 6 \\
\hline 2 & 2 & 3 & 4 & 5 & 3 & 4 & 5 & 6 & 4 & 5 & 6 & 7 \\
\hline 3 & 2 & 4 & 5 & 6 & 4 & 5 & 6 & 7 & 5 & 6 & 7 & 8 \\
\hline 4 & 3 & 5 & 6 & 7 & 5 & 6 & 7 & 8 & 6 & 7 & 8 & 9 \\
\hline 5 & $\overline{4}$ & 6 & 7 & 8 & 6 & 7 & 8 & 9 & 7 & 8 & 9 & 9 \\
\hline \multicolumn{13}{|c|}{ Carga/Força } \\
\hline \multicolumn{2}{|c|}{0} & \multicolumn{4}{|c|}{1} & \multicolumn{3}{|c|}{2} & \multicolumn{4}{|c|}{3} \\
\hline \multicolumn{2}{|c|}{$<5 \mathrm{~kg}$} & \multicolumn{4}{|c|}{5 a $10 \mathrm{~kg}$} & \multicolumn{3}{|c|}{$>10 \mathrm{~kg}$} & \multicolumn{4}{|c|}{$\begin{array}{l}\text { Contração ou rápida } \\
\text { execução de força. }\end{array}$} \\
\hline
\end{tabular}

Fonte: MCATAMNEY; HIGNETT, 2000.

Para a avaliação do pescoço a pontuação varia de 1 a 3, considerando a inclinação e rotação da parte corpórea. O valor de 1 é para inclinação frontal de 20ㅜ, 2 é para inclinação frontal superior a $20^{\circ}$ e para qualquer inclinação para a parte posterior. A pontuação do pescoço pode ser acrescida de 1 quando ocorrer rotação ou inclinação lateral do pescoço (Quadro 2).

A avaliação do posicionamento das pernas varia de 1 a 4 conforme postura de trabalho. Para trabalhos sentados, caminhando ou em pé com apoio bilateral simétrico a pontuação é 1, para a posição de pé apoio unilateral, suporte leve ou postura instável a pontuação é 2 . Ainda no que concerne a pontuação da posição da perna, o posicionamento 
em pé do trabalhador, poderá sofrer um acréscimo de 1 ponto casa haja flexão do joelho de uma ou ambas as pernas de $30^{\circ}$ e $60^{\circ}$ e 2 pontos quando a flexão do joelho for maior do que $60^{\circ}$ (CARDOSO JUNIOR, 2006).

A pontuação do Grupo A pode ser acrescida de mais 3 pontos casa haja carga manipulada ou força exercida ou ainda concentração ou rápida execução de força conforme Tabela 1.

Já os diagramas do Grupo B (Braços, Antebraços e Punhos) são compostos por um total de 36 combinações de posturas, que também resultam num total de 9 pontos encontrados na Quadro 3 que deverão ser adicionados ao ponto de qualidade da pega.

A pontuação do braço é obtida por sua flexão ou extensão, por meio da avaliação do ângulo formado pelo braço e tronco. 0 ângulo semicirculatório de até $20^{\circ}$ a pontuação é 1 , para a elevação do braço para a porte posterior maior de $20^{\circ}$ e elevação frontal maior que $20^{\circ}$ até $45^{\circ}$ a pontuação é 2 , elevação do braço na parte frontal superior a $45^{\circ}$ até $90^{\circ}$ recebe a pontuação 3 e para elevação frontal superior a $90^{\circ}$ a pontuação a ser aplicada 4 (CARDOSO JUNIOR, 2006).

A pontuação para o braço pode aumentar um ponto, se ocorrer elevação ou giro do ombro ou do cotovelo. Caso ocorra apoio de braço para o trabalhador no desenvolvimento da atividade a pontuação diminui um ponto.

O antebraço indica pontuação 1 para flexão entre $60^{\circ}$ e $100^{\circ}$ e dois pontos para flexão inferior a $60^{\circ}$ e superior a $100^{\circ}$.

O punho é pontuado conforme sua flexão medida a partir da postura neutra. Um ponto será aplicado para a pontuação neutra ou com flexão até $15^{\circ}$. Flexão superior a $15^{\circ} \mathrm{a}$ pontuação aplicada é 2. Quando ocorrer desvio radial será acrescido mais um ponto.

No Grupo B deve ser ainda considerar a pega do trabalhador que pode variar de zero a três pontos conforme a condição de trabalho, conforme Quadro 3 (página seguinte).

Os pontos dos Grupos A e B são encontrados pelo cruzamento das pontuações das posturas específicas observadas no Quadro 4 (página seguinte).

Ao valor do cruzamento do Grupo A e B é adiciona pontuação do Grupo C, conforme Quadro 5 (página seguinte). 
Quadro 3 - Pontuações do Grupo B somados ao escore qualidade da pega

\begin{tabular}{|c|c|c|c|c|c|c|c|}
\hline \multirow[b]{2}{*}{ Braço } & \multicolumn{7}{|c|}{ Antebraço } \\
\hline & \multicolumn{4}{|c|}{1} & \multicolumn{3}{|c|}{2} \\
\hline Punho & $\overline{1}$ & 2 & \multicolumn{2}{|c|}{3} & 1 & 2 & 3 \\
\hline 1 & 1 & 2 & \multicolumn{2}{|c|}{3} & 1 & 2 & 3 \\
\hline 2 & 1 & 2 & \multicolumn{2}{|c|}{3} & 2 & 3 & 4 \\
\hline 3 & 3 & 4 & \multicolumn{2}{|c|}{5} & 4 & 5 & 5 \\
\hline 4 & 4 & 5 & \multicolumn{2}{|c|}{5} & 5 & 6 & 7 \\
\hline 5 & 6 & 7 & \multicolumn{2}{|c|}{8} & 7 & 8 & 8 \\
\hline 6 & 7 & 8 & \multirow{2}{*}{\multicolumn{2}{|c|}{$\frac{8}{\mathrm{er}}$}} & 8 & 9 & 9 \\
\hline \multicolumn{7}{|c|}{ Interface } & \\
\hline \multicolumn{2}{|l|}{0 - Bom } & \multicolumn{2}{|c|}{ 1-Aceitável } & \multicolumn{2}{|c|}{2 - Pobre } & \multicolumn{2}{|c|}{3 - Inaceitável } \\
\hline \multicolumn{2}{|c|}{$\begin{array}{l}\text { Boa preensão a } \\
\text { médio alcance, boa } \\
\text { força de agarre. }\end{array}$} & \multicolumn{2}{|c|}{$\begin{array}{l}\text { Preensão aceitável, } \\
\text { mas não ideal ou } \\
\text { interface aceitável via } \\
\text { outra parte do corpo. }\end{array}$} & & $\begin{array}{l}\text { Preensão } \\
\text { inaceitável } \\
\text { apensar de } \\
\text { possível }\end{array}$ & \multicolumn{2}{|c|}{$\begin{array}{l}\text { Desfavorável, pressão } \\
\text { insegura, sem cabos, } \\
\text { manetes. } \\
\text { Interface inaceitável } \\
\text { utilizando outras } \\
\text { partes do corpo. }\end{array}$} \\
\hline
\end{tabular}

Fonte: MCATAMNEY; HIGNETT, 2000.

Quadro 4 - Cruzamentos dos Grupos A e B

\section{Grupo B}

\begin{tabular}{|c|c|c|c|c|c|c|c|c|c|c|c|c|c|}
\hline & & 1 & 2 & 3 & 4 & 5 & 6 & 7 & 8 & 9 & 10 & 11 & 12 \\
\hline \multirow{12}{*}{ 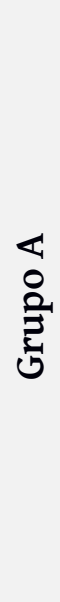 } & 1 & 1 & 1 & 1 & 2 & 3 & 3 & 4 & 5 & 6 & 7 & 7 & 7 \\
\hline & 2 & 1 & 2 & 2 & 3 & 4 & 4 & 5 & 6 & 6 & 7 & 7 & 8 \\
\hline & 3 & 2 & 3 & 3 & 3 & 4 & 5 & 6 & 7 & 7 & 8 & 8 & 8 \\
\hline & 4 & 3 & 4 & 4 & 4 & 5 & 6 & 7 & 8 & 8 & 9 & 9 & 9 \\
\hline & 5 & 4 & 4 & 4 & 5 & 6 & 7 & 8 & 8 & 9 & 9 & 9 & 9 \\
\hline & 6 & 6 & 6 & 6 & 7 & 8 & 8 & 9 & 9 & 10 & 10 & 10 & 10 \\
\hline & 7 & 7 & 7 & 7 & 8 & 9 & 9 & 9 & 10 & 10 & 11 & 11 & 11 \\
\hline & 8 & 8 & 8 & 8 & 9 & 10 & 10 & 10 & 10 & 10 & 11 & 11 & 11 \\
\hline & 9 & 9 & 9 & 9 & 10 & 10 & 10 & 11 & 11 & 11 & 12 & 12 & 12 \\
\hline & 10 & 10 & 10 & 10 & 11 & 11 & 11 & 11 & 12 & 12 & 12 & 12 & 12 \\
\hline & 11 & 11 & 11 & 11 & 12 & 12 & 12 & 12 & 12 & 12 & 12 & 12 & 12 \\
\hline & 12 & 12 & 12 & 12 & 12 & 12 & 12 & 12 & 12 & 12 & 12 & 12 & 12 \\
\hline
\end{tabular}

Fonte: MCATAMNEY; HIGNETT, 2000.

Quadro 5. Incremento de pontuação Grupo C

\begin{tabular}{|l|l|}
\hline Tipo de atividade muscular & Pontuação \\
\hline $\begin{array}{l}\text { Uma ou mais partes do corpo permanecem estáticas por } \\
\text { mais de } 1 \text { minuto }\end{array}$ & +1 \\
\hline $\begin{array}{l}\text { Movimentos repetitivos ocorrem mais de } 4 \text { vezes por } \\
\text { minuto }\end{array}$ & +1 \\
\hline $\begin{array}{l}\text { Alterações posturais importantes ocorrem ou posturas } \\
\text { instáveis são adotadas }\end{array}$ & +1 \\
\hline
\end{tabular}

Fonte: MCATAMNEY; HIGNETT, 2000. 
A pontuação final do REBA é associado à tabela de categorias de ações. 0 Quadro 6 mostra a classificação dos riscos e as categorias de ações.

Quadro 6 - Classificação dos riscos e categoria de ação do método REBA

\begin{tabular}{cccc}
\hline Nível de ação & Escore do REBA & Risco & Ação \\
\hline $\mathbf{0}$ & 1 & Negligenciável & Desnecessária \\
\hline $\mathbf{1}$ & $2-3$ & Baixo & Pode ser necessária \\
\hline $\mathbf{2}$ & $4-7$ & Médio & Necessária \\
\hline $\mathbf{3}$ & $8-10$ & Alto & Necessária breve \\
\hline $\mathbf{4}$ & $11-15$ & Muito alto & Necessária agora \\
\hline Fonte: MCATAMNEY; HIGNETT, 2000. & &
\end{tabular}

\section{RESULTADOS E DISCUSSÃO}

Os resultados das avaliações de ruído e vibração de corpo inteiro realizadas no condutor do transporte urbano foram comparados com os limites de referência e exibidos na Tabela 1 e 2. A Tabela 1 apresenta os dois resultados da avaliação de ruído ocupacional, conforme nível de pressão sonora e a dose de exposição e os horários de avaliação (diurno e vespertino).

Tabela 1. Resultados da avaliação de ruído ocupacional comparados com os limites de referência da NR - 09 e NR 15 e as metodologias de avaliação

\begin{tabular}{cccc}
\hline $\begin{array}{c}\text { Resultado em dB } \\
\text { (A) e Dose (\%) }\end{array}$ & $\begin{array}{c}\text { Metodologia de } \\
\text { avaliação } \\
\text { quantitativa }\end{array}$ & $\begin{array}{c}\text { Norma para } \\
\text { determinação do } \\
\text { limite de referência }\end{array}$ & $\begin{array}{c}\text { Limite de referência } \\
\text { (Limite de tolerância e } \\
\text { nível de ação) }\end{array}$ \\
\hline
\end{tabular}

Diurno: 82,0

$\mathrm{dB}(\mathrm{A}) *$

$65,9 \%$ (dose)

Vespertino: 80,8

$\mathrm{dB}(\mathrm{A}) *$

$55,8 \%(\text { dose })^{*}$
Nível de ação: $80,0 \mathrm{~dB}(\mathrm{~A})$ $50 \%$ dose (nível de ação NR 09) e Limite de tolerância: $85,0 \mathrm{~dB}(\mathrm{~A})$ $100 \%$ dose Limite de tolerância (NR 15)

* Resultado da avaliação projetado para uma jornada padrão de oito horas diárias. Fonte: Autor, 2018.

No que tange o resultado da avaliação de ruído, a intensidade maior avaliada foi de 82,0 dB(A) para uma jornada padrão de oito horas o que aponta uma dose de exposição de 65,9\% obtido no período da manhã. Apesar de inferior ao LT o valor encontrado é superior ao nível de ação de $80 \mathrm{~dB}(\mathrm{~A})$ ou 50\% de dose, sendo necessário o início de ações de controle do risco conforme NR-09 (ATLAS, 2019). 
Em avaliações de ruído, de motorista de ônibus no Município de Rio Branco no Acre realizado por Silva et al (2015), foi observado uma média de intensidade de 86,2 dB (A), indicando intensidade superior ao LT, ampliado pelo ruído da via pública, pois o veículo não possuía sistema de climatização de ar artificial que pudesse manter a janela fechada. Nesse mesmo estudo foi descrito sobre a possibilidade de perda auditiva aos trabalhadores expostos a essa pressão sonora.

Além do risco da perda auditiva Stansfeld e Matheson (2003) descrevem que a exposição a níveis intenso de ruído, pode interferir no desempenho completo da tarefa, modifica o comportamento social, causa incomodo e sugere uma associação com a hipertensão. Neri, Soares e Soares (2005) reforçam que o ruído do trânsito pode também agir contra a saúde mental do profissional, reforçando a necessidade de um controle efetivo.

Para minimização do risco do ruído, Lacerda et al. (2010) sugere a manutenção constante do veículo, redução do tempo de exposição e a realização de exames médicos periódicos. Silva e Correia (2012) descrevem que a ocorrência de níveis elevados de ruído, seriam mais bem solucionados se os projetos construtivos desses coletivos tivessem mais foco no controle desse risco.

No que se refere a avaliação de vibração de corpo inteiro a Tabela 2 exibe os resultados, que estão inferiores os LT da NR 15, para uma jornada de oito horas diárias, dividindo-os em aceleração $\left(m \cdot s^{-2}\right)$ e valor de dose de vibração $\left(m \cdot s^{-1,75}\right)$.

Tabela 2. Resultados da avaliação de vibração ocupacional comparados com os limites de referência da NR 09 e NR 15.

\begin{tabular}{cccc}
\hline $\begin{array}{c}\text { Resultado } \\
\text { em m.s } \mathbf{s}^{-2} \text { em Dose } \\
\text { (VDV) }\end{array}$ & $\begin{array}{c}\text { Metodologia } \\
\text { de avaliação } \\
\text { quantitativa }\end{array}$ & $\begin{array}{c}\text { Norma para } \\
\text { determinação do } \\
\text { limite de } \\
\text { referência }\end{array}$ & $\begin{array}{c}\text { Limite de referência } \\
\text { (Limite de tolerância } \\
\text { e nível de ação) }\end{array}$ \\
\hline $0,47 \mathrm{~m} \cdot \mathrm{s}^{-2 *}$ e $10,20 \mathrm{~m} \cdot \mathrm{s}^{-1,75 *}$ & NHO 09 & NR 09 e NR 15 & $\begin{array}{l}\text { Nível de ação: } 0,5 \mathrm{~m} \cdot \mathrm{s}^{-2} \\
\text { e } 9,1 \mathrm{~m} \cdot \mathrm{s}^{-1,75} \\
\text { Limite de tolerância: } \\
1,1 \mathrm{~m} \cdot \mathrm{s}^{-2} \text { e } 21,0 \mathrm{~m} \cdot \mathrm{s}^{-1,75}\end{array}$ \\
& & \\
\hline $\begin{array}{l}\text { * Resultado da avaliação projetado para uma jornada padrão de oito horas diárias. } \\
\text { Fonte: Autor, 2018. }\end{array}$
\end{tabular}

A avaliação de vibração de corpo inteiro não apresentou risco ao observamos na ótica do LT da NR 15 anexo 8, porém a intensidade da dose de vibração superou o nível de ação, indicando a necessidade de iniciar a adoção das medidas e controle (ATLAS, 2019). 
Figueiredo, Silva e Barnabé (2016) em avaliação de motoristas de ônibus, obtiveram intensidade similar aos dessa pesquisa, com valores superiores ao nível de ação e inferiores ao LT, adotado como referência a Diretiva Europeia número 44 de 2002 (DIRETIVA EUROPEIA, 2002) que possui valores similares aos da NR - 15 anexo 8.

Balbinot e Tamagna (2002) ao realizarem as avaliações de vibração de corpo inteiro, em motoristas de ônibus de coletivo urbano na cidade de Porto Alegre no sul do Brasil, conforme norma International Organization for Standardzation - ISO 2631/1997, identificaram intensidade superior ao sugerido nessa normativa para conforto e indicaram em seus resultados que os assentos dos ônibus não apresentavam comportamento dinâmico adequado. Resultados esses similares obtidos por Walber (2009) em passageiros de coletivos urbanos intermunicipais.

Assunção e Pimenta (2015), em seu estudo, sugerem considerar que a exposição ocupacional à vibração de corpo inteiro pode ter relação com a hipertensão de motoristas e Silva e Mendes (2005) descrevem que esse risco pode causar efeitos adversos à coluna vertebral como degeneração precoce da região lombar, lombalgia e hérnia de disco, segundo os tópicos mais recorrentes na literatura sobre o tema.

Ribas et al. (2014) sugere para minimização do risco de vibração de corpo inteiro a manutenção periódica do veículo ou a instalação de novos mecanismos de suspensão veicular. Enquanto Balbinot. Tamagna (2002) e Walber (2009) sugerem adequação dos assentos em coletivos urbanos, diante o risco vibração, para maior conforto do motorista.

Para avaliação ergonômica foi verificado que o motorista ao dirigir mantém sua coluna na posição neutra. Ao acionar os pedais de embreagem, acelerador e freio, o corpo perde o alinhamento, pois é necessário a flexão dos joelhos e do pé, mesmo estando próximo aos pedais. No membro superior o motorista realiza o trabalho repetitivo de manuseio do câmbio e condução do veículo (Figura 2). 
Figura 2. Posicionamento do motorista no posto de trabalho.

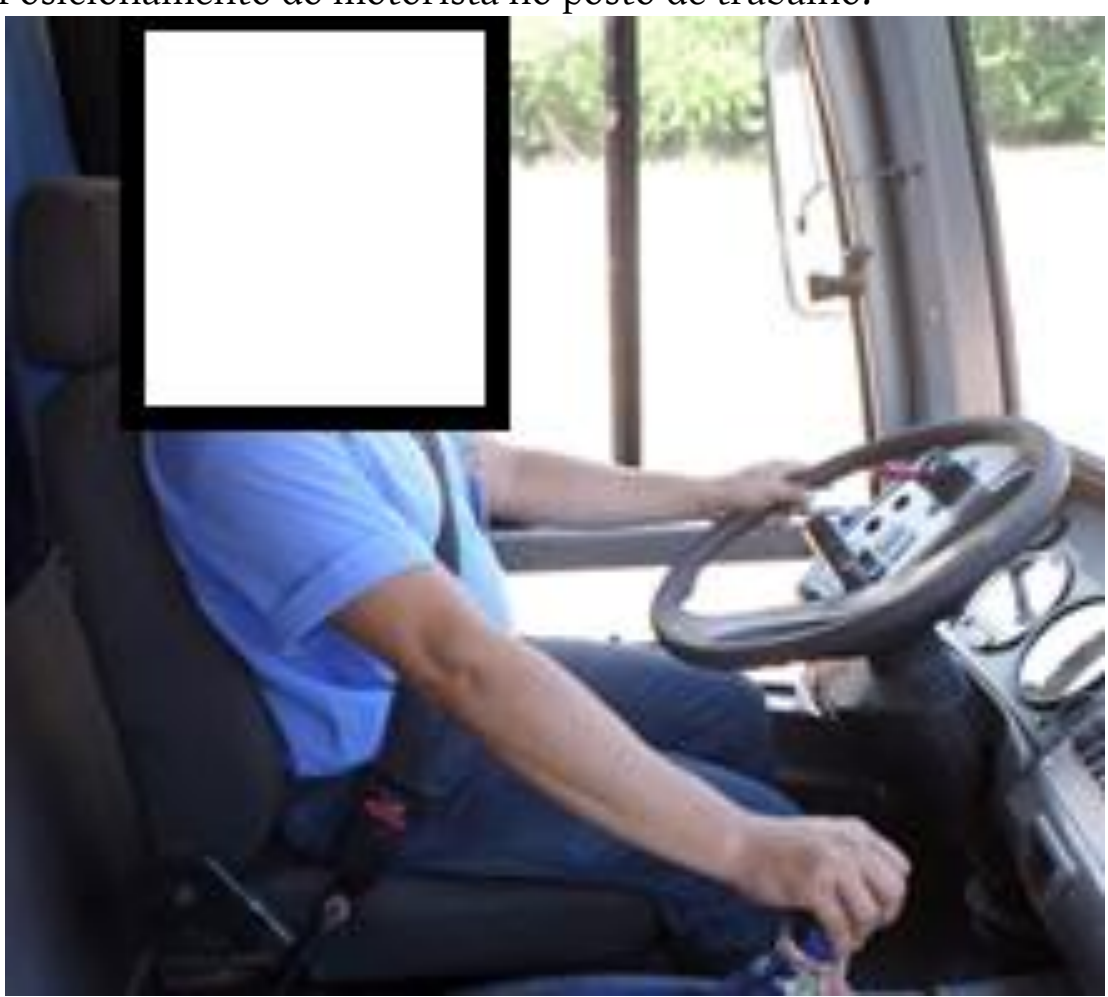

Fonte: Autor, 2018.

A avaliação da postura de trabalho foi realizada conforme REBA e seu resultado apresentado na Tabela 3.

Tabela 3. Avaliação da postura do motorista conforme REBA

\begin{tabular}{|c|c|c|c|}
\hline Grupo & Membro & Postura & Pontuação \\
\hline \multirow{3}{*}{$\begin{array}{l}\text { A (Tronco, pescoço } \\
\text { e pernas) }\end{array}$} & Tronco & Flexão entre $0^{\circ}$ e $20^{\circ}$ & 2 \\
\hline & Pescoço & Flexão entre $0^{\circ}$ e $20^{\circ}$ & 1 \\
\hline & Pernas & Sentado. & 1 \\
\hline \multicolumn{3}{|l|}{ Grupo A } & 1 \\
\hline \multirow[t]{3}{*}{$\begin{array}{l}\text { B (Braço, antebraço } \\
\text { e punho) }\end{array}$} & Braço & $\begin{array}{l}\text { Flexão maior de } 20^{\circ} \text { e } \\
\text { inferior a } 45^{\circ} \text {. Acrescido } \\
\text { um ponto devido ao } \\
\text { afastamento do braço } \\
\text { em relação ao corpo }\end{array}$ & $2+1=3$ \\
\hline & Antebraço & Flexão entre $60^{\circ}$ e $100^{\circ}$ & 1 \\
\hline & Punho & Posição neutra & 1 \\
\hline \multicolumn{3}{|l|}{ Grupo B } & 3 \\
\hline \multirow{2}{*}{$\begin{array}{l}\text { C (Incremento da } \\
\text { pontuação por } \\
\text { atividade muscular) }\end{array}$} & \multicolumn{2}{|c|}{$\begin{array}{l}\text { Uma ou mais partes do corpo permanecem } \\
\text { estáticas por mais de } 1 \text { minuto }\end{array}$} & 1 \\
\hline & \multicolumn{2}{|c|}{$\begin{array}{l}\text { Movimentos repetitivos ocorrem mais de } 4 \\
\text { vezes por minuto }\end{array}$} & 1 \\
\hline \multicolumn{3}{|c|}{ Pontuação conforme relação do Grupo A e B } & 2 \\
\hline \multicolumn{3}{|l|}{ Grupo C } & 2 \\
\hline \multicolumn{3}{|c|}{ Pontuação final total REBA } & 4 \\
\hline
\end{tabular}

Fonte: Autor, 2018. 
O resultado da avaliação ergonômica apresentou pontuação do REBA igual a 4 (risco médio) indicando a necessidade de ações para melhorar as condições de trabalho. A avaliação retrata também a reclamação do trabalhador, que segundo o mesmo é frequente dores no pé e no joelho esquerdo e no cotovelo.

Em avaliação de posto de trabalho similar, no município de Sucre na Bolívia, Flórez, Barreto e Jiménez (2017) obtiveram dados de avaliação ergonômica no método REBA mais críticos do que os obtidos nessa pesquisa. A diferença deve-se ao fato, que em Sucre, é exigida uma torção lateral do tronco do condutor, que não foi observada nos condutores do Pará.

Neri, Soares e Soares (2005) sugere às empresas de transporte rodoviário urbano a adoção do câmbio automático nos veículos de transporte urbano, para minimizar o risco ergonômico na qual o motorista está exposto, pois o mesmo chega a realizar a troca de marcha mais de mil vezes por jornada de trabalho, exigindo uma mudança de postura constante que causa fadiga.

\section{CONCLUSÕES}

Diante dos resultados apresentados é possível identificar que os riscos físicos ruído $(82,0 \mathrm{~dB}(\mathrm{~A})$ e $80,08 \mathrm{~dB}(\mathrm{~A}))$ e vibração $\left(0,47 \mathrm{~m} \cdot \mathrm{s}^{-2}\right.$ e $\left.10,20 \mathrm{~m} \cdot \mathrm{s}^{-1,75}\right)$ constante no ambiente de trabalho obtiveram valores de avaliação inferior ao limite de tolerância, porém superior ao nível de ação, conforme normativas nacionais de referência.

Pesquisas sugerem, para a minimização do risco de ruído e a proteção ao trabalhador, projetos construtivos de coletivos mais adequados, a manutenção periódica do veículo, redução do tempo de exposição e a realização exames periódicos no trabalhador. Para a redução da exposição à vibração ocupacional de corpo inteiro segure-se a manutenção periódica do veículo ou até mesmo a alteração do sistema de suspensão veicular e assentos com comportamento dinâmico mais adequados, que podem contribuir para um melhor bem-estar do trabalhador exposto.

A avaliação ergonômica segundo critério Rapid Entire Body Assessment foi classificada como risco médio, sendo necessário estudos para intervenção nas atividades. Pode se contribuir com a tarefa o emprego de câmbio automático nos veículos de transporte urbano, 
que reduziria o afastamento do braço ao corpo para mudança de marcha de forma repetitiva.

O transporte coletivo urbano com sua relevância social e econômica, também deve ser observado o seu interior dos veículos, local esse em que os motoristas despendem grande parte do seu dia e necessitam de ambientes mais seguros, confortáveis e modernos, compatíveis com a relevância do seu trabalho.

\section{REFERÊNCIA}

ALQUIMIM, A. F.; BARRAL, A. B. C. R.; GOMES, C.; REZENDE, M. C de. Avaliação dos fatores de risco laborais e físicos para doenças cardiovasculares em motoristas de transporte urbano de ônibus em Montes Claros (MG). Ciência \& Saúde Coletiva, v. 17, p. 2151-2158, 2012.

Anuário Estatístico de Acidentes do Trabalho: AEAT 2017 / Ministério da Fazenda ... [et al.]. - vol. 1 (2009) DATAPREV. - Brasília: MF, 2017. 996 p. Anual. ISSN 1676-9694.

ASSUNÇã̃o, A. A.; PIMENTA, A. M. A exposição à vibração e a hipertensão arterial em trabalhadores do transporte coletivo metropolitano. Revista Brasileira de Saúde Ocupacional, v. 40, n. 132, 2015.

ATLAS, MANUAL DE LEGISLAÇ̃̃o. Segurança e medicina do trabalho. 74. ed. São Paulo: Atlas SA, 2019.

BALBINOT, A.; TAMAGNA, A. Avaliação da transmissibilidade da vibração em bancos de motoristas de ônibus urbanos: um enfoque no conforto e na saúde. Revista brasileira de engenharia biomédica. Rio de Janeiro, RJ. Vol. 18, n. 1 (jan./abr. 2002), p. 31-38, 2002.

BATTISTON, N.; CRUZ, R. M.; HOFFMANN, M. H. Condições de trabalho e saúde de motoristas de transporte coletivo urbano. Estudos de Psicologia, V. 11, n. 3, p. 333-343, 2006.

CARDOSO JUNIOR, M. M. C. Avaliação ergonômica: Revisão dos métodos para avaliação postural. Revista produção online, v. 6, n. 3, 2006.

Diretiva Europeia. Relativa às prescrições mínimas de segurança e saúde respeitantes à exposição dos trabalhadores aos riscos devidos aos agentes físicos (vibrações). Parlamento Europeu; Conselho da União Europeia. (2002). Directiva 2002/44/CE do Parlamento Europeu e do Conselho, de 25 de junho de 2002.

FIGUEIREDO, M. A. de M.; SILVA, L. F.; BARNABÉ, T. L. Transporte coletivo: vibração de corpo-inteiro e conforto de passageiros, motoristas e cobradores. Journal of Transport Literature, v. 10, n. 1, p. 35-39, 2016.

FLÓREZ, C. M. P.; BARRETO, M. C. B.; JIMÉNEZ, J. M. P. Riesgo ergonómico en los conductores de transporte intermunicipal de Sucre. Universidad-Ciencia-Innovación y Sociedad: Desde la Perspectiva Laboral, p. 49. 2017.

Fundação Amazônia de Amparo a Estudos e Pesquisas - FAPESPA. Estatísticas Municipais Paraenses: Almeirim. / Diretoria de Estatística e de Tecnologia e Gestão da Informação. - Belém, 2016. 58f.: il. Semestral, n. 1, jul. / dez.

FUNDACENTRO - FUNDAÇÃO JORGE DUPRAT FIGUEIREDO, DE SEGURANÇA E MEDICINA DO TRABALHO. Norma de Higiene Ocupacional NHO 01: procedimento técnico para a avaliação da exposição ocupacional ao ruído contínuo ou intermitente e impacto. São Paulo - SP. FUNDACENTRO. 2001. 40 p. 
FUNDACENTRO - FUNDAÇÃO JORGE DUPRAT FIGUEIREDO, DE SEGURANÇA E MEDICINA DO TRABALHO. Norma de Higiene Ocupacional NHO 09: avaliação da exposição ocupacional a vibrações de corpo inteiro: procedimento técnico. São Paulo-SP: Fundacentro, 2013. 63 p.

LACERDA, A.; FIGUEIREDO, G.; MASSAROLO NETO, J., MARQUES, J. M. Achados audiológicos e queixas relacionadas à audição dos motoristas de ônibus urbano. Revista da Sociedade Brasileira Fonoaudiologia, v. 15, n. 2, p. 161-6, 2010.

MATOS, S. L. de; NEVES, F. S. Avaliação do estresse dos motoristas da empresa Viação Javaé de Gurupi/TO. Revista Desafios, v. 1, n. 2, p. 185-197, 2015.

MCATAMNEY, L. Y. N. N.; HIGNETT, S. REBA: Rapid Entire Body Assessment. Applied ergonomics, v. 31, p. 201205, 2000.

MORAES, B. C. de. CostA, J. M. N. da. COSTA, A. C. L. da. cosTA, M. H. Variação espacial e temporal da precipitação no estado do Pará. Acta Amazon, v. 35, p. 207-217, 2005.

MORAES, R. de C. M. de; BORDALO, C. V. de S.; MEYER, E. A.; SILVA, U. R. da; LIMA, G. G. de. DAMASCENO, L. de O.; CARDOSO, M. dos S. Qualidade do Transporte Urbano da Região Metropolitana de Belém. Revista Científica Multidisciplinar Núcleo do Conhecimento. Ano 03, Ed. 02, Vol. 03, pp. 147-156, Fevereiro de 2018. ISSN: 2448-0959

NERI, M.; SOARES, W. L.; SOARES, C. Condições de saúde no setor de transporte rodoviário de cargas e de passageiros: um estudo baseado na Pesquisa Nacional por Amostra de Domicílios. Cadernos de Saúde Pública, v. 21, p. 1107-1123, 2005.

RIBAS, R. L.; SCHLOSSER, J. F.; FRANTZ, U. G.; FARIAS, M. S. de.; RODRIGUES, F. A. Exposição humana à vibrações de corpo inteiro em um trator agrícola com pneus radiais. Ciência Rural, v. 44, n. 9, 2014.

SILVA, L. F.; MENDES, R. Exposição combinada entre ruído e vibração e seus efeitos sobre a audição de trabalhadores. Revista Saúde Pública, São Paulo, v. 39, n. 1, p. 9-17, Jan. 2005.

SILVA, L. F.; CORREIA, F. N. Avaliação da exposição de passageiros ao ruído no interior de ônibus do transporte público do munícipio de Itajubá. Revista CEFAC, v. 14, n. 1, 2012.

SILVA, J. A. B. da; SILVA, S. da. Critério de qualidade em serviços de transporte público urbano: Uma contribuição teórica. Brazilian Journal of Production Engineering. São Mateus, Vol. 4, n.1, p. 83 - 98. (2018). Editora CEUNES/DETEC.

SILVA, R. P. M.; SANCES, C. G. F.; FERREIRA, C. R. T.; SCHIMER, J.; RODRIGUES, P. F. Níveis de intensidade sonora no transporte coletivo urbano de Rio Branco-AC. Journal of Amazon Health Science, v. 1, n. 1, p. 82-87, 2015.

STANSFELD, S. A.; MATHESON M. P. Noise pollution: non-auditory effects on health. British medical bulletin, v 68, n 1, p. 243-257, 2003.

WALBER, M. Avaliação dos níveis de vibração existentes em passageiros de ônibus rodoviários intermunicipais, análise e modificação projetual. Tese de doutorado (Programa de pós-graduação em engenharia mecânica) Universidade Federal do Rio Grande do Sul. 199 f. 2009.

\section{$(\mathbf{c c})$ Br}

Este trabalho está licenciado com uma Licença Creative Commons - Atribuição 4.0 Internacional. 